\title{
"POTATOPLASTIC": Desenvolvimento de bioplásticos a partir dos subprodutos da indústria de batata frita
}

\author{
Idalina Gonçalves ${ }^{a, b,}$, Ana Barrab, Joana Lopes ${ }^{b}$, Cláudia Nunes ${ }^{a, b}$, Paula Ferreira ${ }^{b}$, Manuel A. Coimbra ${ }^{a}$ \\ aOPNA, Departamento de Química, Universidade de Aveiro

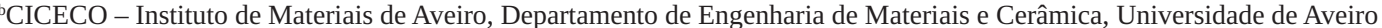 \\ idalina@ua.pt
}

\begin{abstract}
“POTATOPLASTIC”: Development of bioplastics using potato chips byproducts - The environmental impact caused by plastic waste accumulation has led to the search for biodegradable materials, such as bioplastics. Plastics are materials that can be molded. When produced from renewable resources, as biomass, they are named bioplastics. Despite of its commercial existence, the industrial production is still reduced due to unsuitable mechanical and physicochemical properties, namely restricted tensile strength and/or flexibility, moisture sensitivity and limited barrier properties, as well as costs that are not yet competitive with those of plastics. As a potential strategy to overcome these constraints and promote bioplastics expansion, "POTATOPLASTIC" project was designed, supported by Portugal2020 program, aiming the valorization of potato chips industry byproducts through the recovery of compounds suitable for bioplastics production. In this project, starch, oil, and waxes from potato washing waters, frying residues, and potato peels, respectively, were extracted. The combination of starch native and/or modified with oils and/or waxes allows to handle materials characteristics, yielding transparent, flexible and hydrophobic films with potential application as food packaging. This project promotes the sustainable development of bioplastics, creating the basis for translating the methodology into an industrial scale in the near future.
\end{abstract}

O impacto ambiental causado pela acumulação de resíduos plásticos tem levado à procura de materiais biodegradáveis, como os bioplásticos. Os plásticos são materiais com capacidade de serem moldados. Quando produzidos a partir de fontes renováveis, como a biomassa, são designados bioplásticos. Apesar da sua existência comercial, a produção a nível industrial é ainda reduzida devido às propriedades mecânicas e físico-químicas, nomeadamente restrita resistência à rutura e/ou flexibilidade, sensibilidade a condições de humidade e limitadas propriedades de barreira, assim como os custos, que ainda não são competitivos com os dos plásticos. Como potencial estratégia para colmatar estas limitações e promover a expansão dos bioplásticos, surge o projeto "POTATOPLASTIC", financiado através do programa de apoio Portugal2020, que visa a valorização dos subprodutos da indústria de batata frita através da recuperação de compostos adequados à produção de bioplásticos. Neste projeto, o amido, o óleo e as ceras foram extraídos, respetivamente, das águas de lavagem, dos resíduos de fritura e das cascas de batata. A combinação do amido nativo e/ou modificado com óleo e/ou ceras permite manipular as características dos materiais, obtendo-se películas transparentes, flexíveis e hidrofóbicas com potencial aplicação como embalagem alimentar. Este projeto promove o desenvolvimento sustentável de bioplásticos, criando a base para que, num futuro próximo, se traduza numa metodologia transponível à escala industrial.

\section{Bioplásticos}

Grande parte dos plásticos com que lidamos no nosso dia a dia são polímeros obtidos a partir de monómeros derivados de recursos fósseis. Existem vários setores económicos, tais como a indústria eletrónica, automóvel, construção civil e produtora de embalagens que utiliza plásticos durante a sua atividade, dando origem a grandes quantidades de resíduos plásticos. O descarte destes resíduos pode ser realizado através de quatro vias: despejo em aterro, incineração, reciclagem ou biodegradação. Contudo, apenas as duas primeiras opções são possíveis para todos os tipos de plásticos. A incineração de plástico promove a formação de dióxido de carbono, que contribui para o efeito de estufa, e de compostos orgânicos parcialmente oxidados, poluentes atmosféricos, tais como os hidrocarbonetos aromáticos policíclicos (PAHs), classificados no Grupo 2B pela Agência Internacional de Investigação em Cancro (IARC) como potencialmente cancerígenos, e as dioxinas, que são poluentes persistentes. Da incineração resultam também cinzas contendo metais que, pela sua toxicidade, são uma fonte de contaminação para os recursos hídricos subterrâneos subjacentes aos aterros utilizados para a sua eliminação [1]. Assim sendo, geralmente, os resíduos plásticos são maioritariamente depositados em aterro, o que não é de todo sustentável, dada a ocupação de grandes áreas territoriais, diminuindo os recursos terrestres adequados para outros fins de maior valor social. Muitos destes resíduos acabam sendo direcionados para os oceanos. Como estes materiais não são biodegradáveis, ou seja, não são degradados por agentes biológicos naturais, a sua acumulação no meio ambiente gera consequências ambientalmente adversas, incluindo a produção de compostos orgânicos xenobióticos, tais como os compostos alifáticos halogenados, hidrocarbonetos aromáticos e até mesmo compostos com efeito pesticida, de acordo com a origem dos resíduos plásticos descartados [2]. De modo a corrigir estes fenómenos de poluição, é necessário desenvolver alternativas sustentáveis derivadas de fontes renováveis e, portanto, biodegradáveis [3]. Neste sentido, os bioplásticos têm sido objeto de estudo ao nível académico e industrial. A produção destes materiais é ambientalmente aceitável, 
uma vez que utiliza matérias-primas renováveis, normalmente biopolímeros, que durante a sua manipulação, geralmente não necessitam de elevada energia e não dão origem a produtos de reação tóxicos [4]. Na tabela 1 encontram-se alguns exemplos de bioplásticos, que inclui o ácido polilático (PLA), os poli-hidroxialcanoatos (PHAs), o amido e os ésteres de celulose, frequentemente utilizados na produção de filmes com aplicação agrícola, em utensílios de cozinha descartáveis e em embalagens alimentares.

Apesar de serem materiais ecológicos, os bioplásticos ainda apresentam, para a maioria das aplicações, propriedades mecânicas de qualidade inferior quando comparadas com os plásticos obtidos à base de petróleo. O desenvol- vimento de materiais compósitos, como por exemplo, o reforço de formulações à base de amido com outros biopolímeros ou fibras provenientes da biomassa lenhocelulósica, ou até mesmo a produção de bioplásticos híbridos, cuja formulação apresenta componentes de origem biológica e também de origem petroquímica, melhoram as propriedades mecânicas do material. Contudo, quanto mais complexa for a formulação de um bioplástico, mais dispendioso será o seu processo de produção, pois diferentes consumos de energia, solventes, metodologias de processamento e estratégias de seleção dos produtos da reação (purificação) serão utilizados, o que inviabiliza o desenvolvimento de soluções sustentáveis e posterior transposição à escala industrial.

Tabela 1 - Nomes, estruturas químicas e exemplos dos bioplásticos mais relevantes.

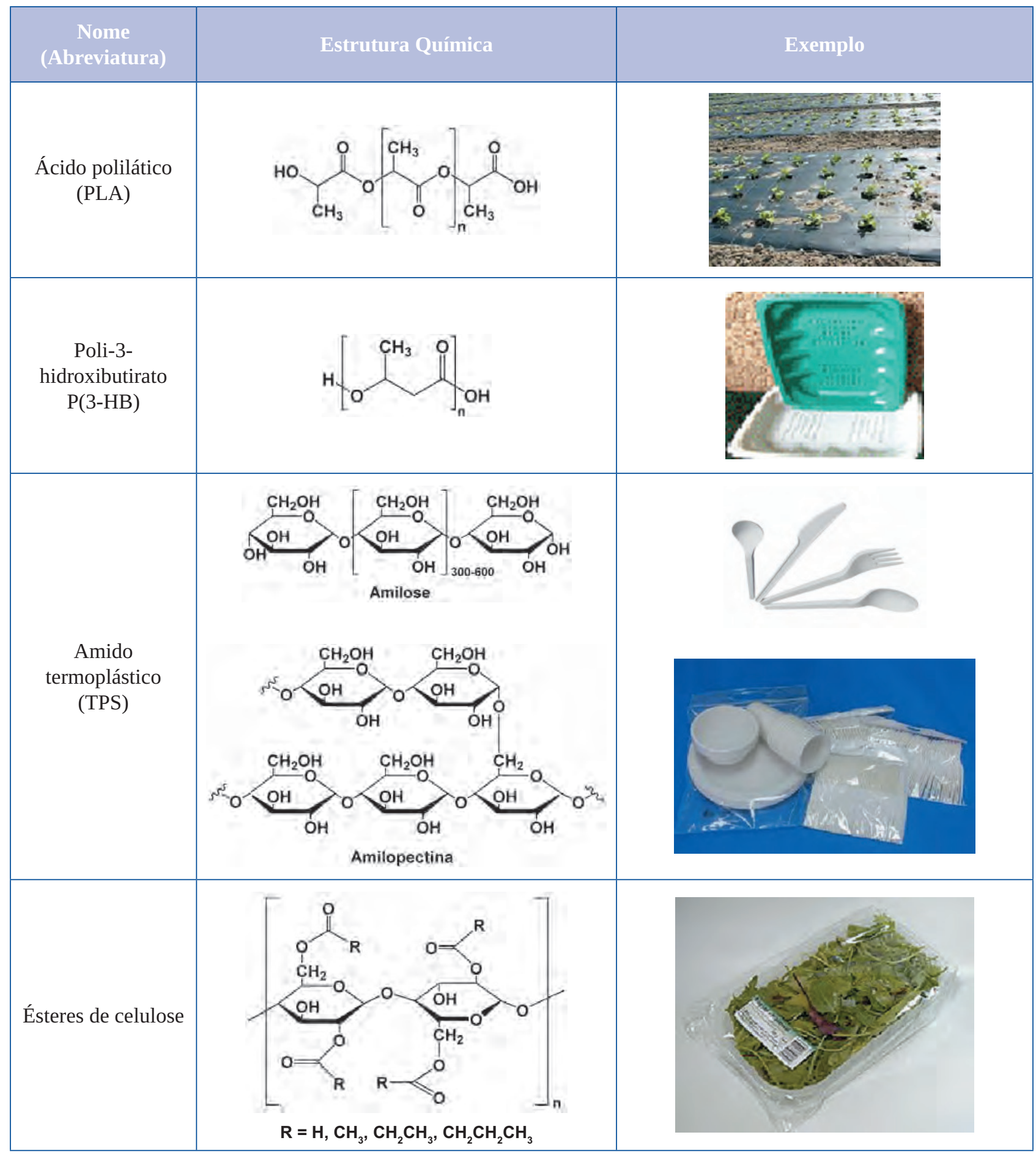


Resíduos orgânicos - uma fonte de matérias-primas para a produção sustentável de bioplásticos

Os bioplásticos podem ser produzidos por: (i) utilização de matérias-primas primárias, cuja cultura é especialmente destinada ao desenvolvimento de bioplásticos, tais como milho, arroz, batata e trigo; (ii) processamento de matérias-primas secundárias, como os resíduos orgânicos provenientes da atividade agroalimentar; e (iii) uso de matérias-primas de origem fóssil [5]. Atualmente, o processamento de matérias-primas secundárias tem um valor socioeconómico acrescido, pois estas deixam de ser consideradas resíduos e passam a ser consideradas subprodutos ou mesmo coprodutos de um determinado processo industrial, sendo o seu custo, enquanto matéria-prima, menor do que o uso de matérias-primas primárias. A esta reutilização dos resíduos atribui-se o nome de economia circular. Ao contrário da atual economia tendencialmente linear, em que as matérias-primas são extraídas, transformadas em produtos (etapa que também gera resíduos não aproveitados) para posteriormente serem comercializados e, após a devida utilização, serem simplesmente descartados, a economia circular defende que os resíduos sejam transformados em subprodutos ou coprodutos que promovam novas utilizações através da recuperação e reciclagem. Quando aplicada aos setores da indústria agroalimentar e dos bioplásticos, a economia circular potencia o desenvolvimento de processos sustentáveis, evitando a utilização de materiais de origem petroquímica e a competição com o consumo dos alimentos.

Entre os vários resíduos orgânicos provenientes da atividade agroalimentar encontram-se, por exemplo, desperdícios de frutas e vegetais, carnes e seus derivados, óleos e biomassas celulósicas, dos quais podem ser recuperados compostos fenólicos, carotenoides, lípidos, proteínas, ácidos gordos esterificados, fitoesteróis, amido, entre outros compostos. [6]. Nesta panóplia existem várias matérias-primas que podem ser conjugadas, dando origem a novos materiais bioplásticos.

\section{O Projeto POTATOPLASTIC}

No âmbito da implementação da economia circular entre diversos setores industriais, surge o projeto "POTATOPLASTIC", financiado através do programa de apoio Portugal2020, visando a valorização dos resíduos da indústria de batata frita como potenciais subprodutos para o desenvolvimento de compostos bioplásticos. Este projeto consiste numa colaboração entre a "Isolago" (Pontével, Cartaxo), cuja atividade incide na produção de materiais plásticos e a Universidade de Aveiro, tendo a empresa de batata frita "A Saloinha” (Mafra) como fornecedora da matéria-prima. Da indústria de batata frita resultam vários resíduos (águas de lavagem, cascas de batata, fragmentos que resultam do corte da batata, batatas com defeito não destinadas ao consumo humano e resíduos derivados dos processos de fritura) que são geralmente desperdiçados. Apenas entre 2013 e 2016, a “A Saloinha” produziu, em média, cerca de 750000 L/ano de águas de lavagem de batata e $337700 \mathrm{~kg}$ de resíduos de batata crua e cascas. Este fluxo de subprodutos representa um esforço significativo na gestão de resíduos, o que contribui negativamente para a sustentabilidade da sua atividade económica. Para a valorização destes subprodutos, com base na composição química da batata, estão a ser recuperados os resíduos existentes na empresa "A Saloinha" e aplicados no desenvolvimento de bioplásticos. Deste modo, os resíduos deixam de ser simplesmente eliminados e passam a ser considerados subprodutos que poderão ser introduzidos numa outra cadeia de produção.

\section{Os subprodutos da indústria de batata frita como fontes de matérias-primas}

Na figura 1 apresentam-se os subprodutos recuperados da indústria de batata frita, nomeadamente águas de lavagem, cascas de batata e resíduos de fritura. Destes subprodutos foram recuperados amido, ceras e óleo, respetivamente, sendo o amido o componente maioritário.

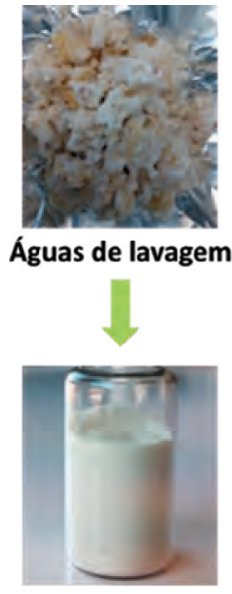

Amido

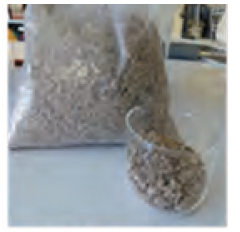

Cascas de batata

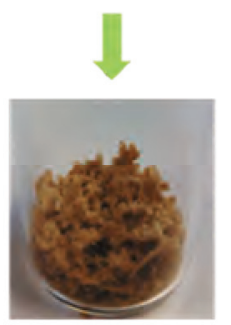

Ceras

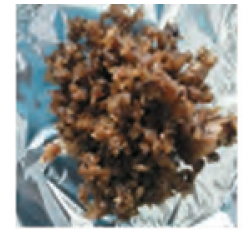

Resíduos de fritura

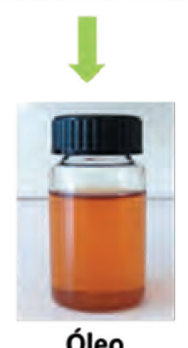

Óleo
Figura 1 - Matérias-primas recuperadas dos subprodutos provenientes da empresa de batata frita "A Saloinha” com vista à elaboração de bioplásticos.

$\mathrm{O}$ amido encontra-se nas células vegetais sob a forma de grânulos cujo tamanho pode variar ente 2 e 150 m, de acordo com a fonte botânica. O tamanho do grânulo do amido da batata pode variar entre os 5 e $100 \mu \mathrm{m}$ [7]. A amilose e a amilopectina são os dois polissacarídeos que constituem o amido. A amilose é uma macromolécula que apresenta entre 300 e 600 resíduos de D-glucopiranose, ligados por ligações glicosídicas $\alpha-1,4$, e que confere às partes lineares dos polissacarídeos componentes do amido uma estrutura helicoidal. Por sua vez, a amilopectina é constituída por cerca de 1400 resíduos de D-glucopiranose ligados por ligações $\alpha-1,4$ e alguns desses resíduos ramificados com ligações $\alpha-1,6$. A razão amilose/amilopectina varia de acordo com a origem do amido [8]. Quando utilizado na preparação de bioplásticos, este parâmetro influencia as propriedades físico-químicas e funcionais do amido, pois quanto maior a quantidade de amilose, maior a resistência ao processo de hidratação dos grânulos, o que dificulta o fenómeno de gelatinização [9].

Quando aplicado na produção de materiais, o amido confere sempre características hidrofílicas, o que limita a sua aplicação. Como alternativa surge, por exemplo, a combinação do amido com outros biopolímeros de cariz hidrofóbico. É neste contexto que surge a recuperação de ceras das cascas de batata e de óleo dos resíduos de fritura 
para incorporação nas formulações dos materiais à base do amido derivado das águas de lavagem. As ceras cuticulares, constituídas por poliésteres reticulados de ácidos e álcoois gordos, como a cutina e a suberina, e que podem ser extraídas com solventes orgânicos, encontram-se nas células da epiderme da batata, e conferem propriedades de barreira contra as perdas de água e a entrada de bactérias ou fungos (Tabela 2), e, consequente, caráter lipofílico [10,11]. Por sua vez, o óleo possui triacilgliceróis, com ácidos gordos com uma cadeia carbonada de 14 a 18 átomos de carbono (Tabela 2). Quando incorporadas em matrizes poliméricas hidrofílicas, como os materiais à base de amido, estas frações lipídicas permitem diminuir a sua hidrofilicidade, ajustando a capacidade de resistência aos meios aquosos. filicidade destes materiais, a incorporação de ceras e óleo recuperados dos subprodutos da indústria de batata frita nos filmes à base de amido deve ser estudada para várias razões amido/óleo e amido/ceras.

\section{Propriedades dos filmes}

A figura 2 demonstra que a combinação do amido com óleo e/ou ceras deu origem a películas transparentes, flexíveis e hidrofóbicas, com alteração das propriedades mecânicas dos filmes, nomeadamente da tensão à tração, da capacidade elástica (módulo de Young) e do alongamento de cada material, determinadas de acordo com a norma ASTM D 882-83.

A incorporação de óleo conduziu à produção de materiais com maior rigidez (visível pelo aumento do módulo

Tabela 2 - Constituintes das ceras e do óleo extraído das cascas e dos resíduos de fritura provenientes da indústria de batata frita.

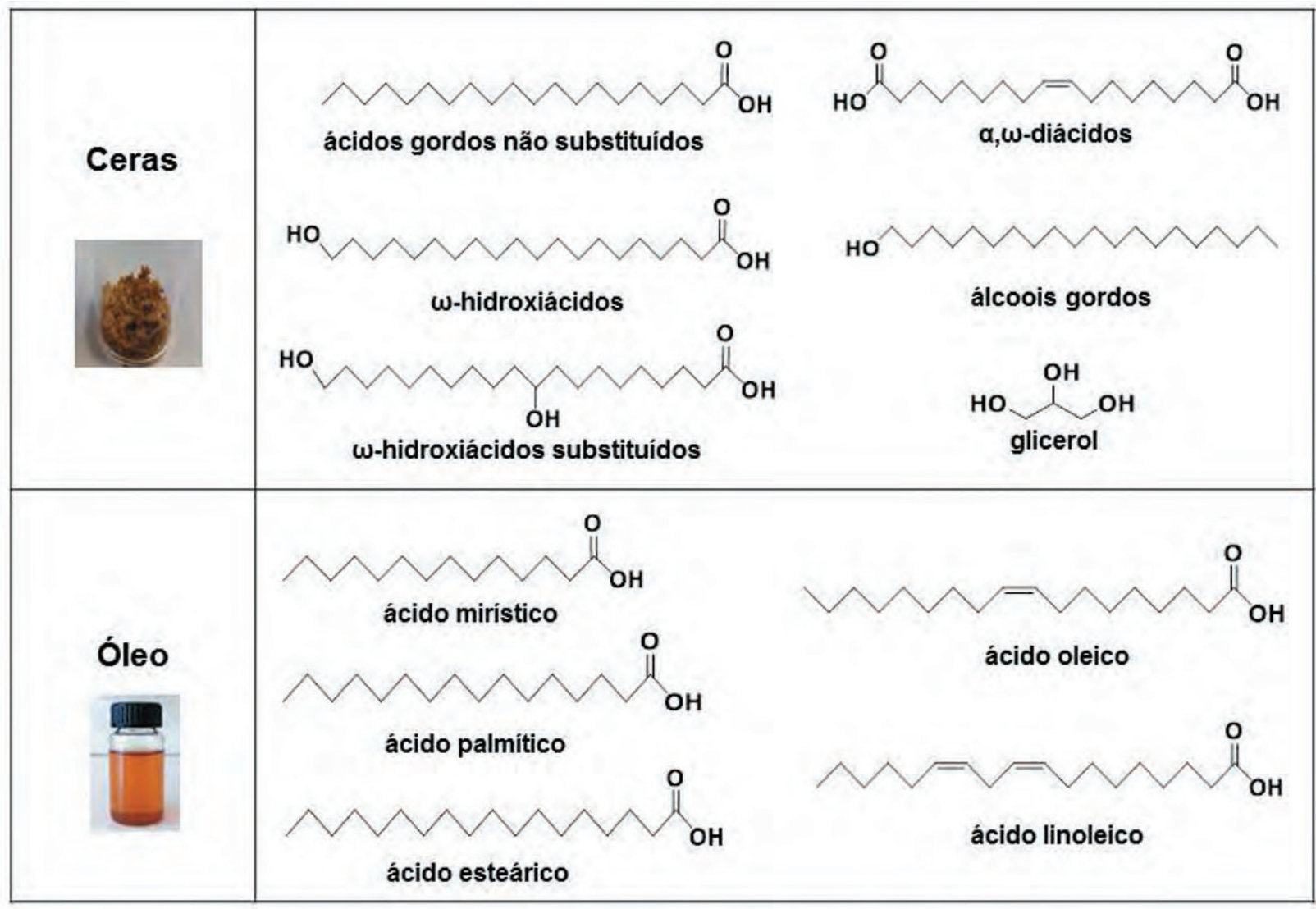

Preparação de filmes à base de amido, óleo e ceras recuperados

Os filmes à base de amido podem ser preparados por um processo de gelatinização seguido da evaporação do solvente. Após a dispersão dos grânulos de amido em água destilada é adicionado glicerol, um agente plasticizante, e aquece-se a suspensão, dando origem à formação de um gel. Após um processo de filtração sob vácuo para remover qualquer impureza remanescente dos subprodutos, o gel é ultra-agitado, com o auxílio de um dispersor homogeneizador, frequentemente designado por ultraturrax, e vertido em placas de acrílico (o molde escolhido para a preparação dos filmes). Este é submetido a um processo de secagem em estufa a baixa temperatura, do qual resulta uma película, vulgarmente designada por filme. Para modular a hidro- de Young) do que as formulações apenas à base de amido. A adição de óleo complementa o efeito plasticizante do glicerol, também incluído na formulação. Por sua vez, a adição de ceras reflete-se num aumento de rigidez, especialmente para elevadas quantidades. O aumento da capacidade de deformação dos filmes à base de amido sugere que os compostos lipídicos conduzem à disrupção das interações entre as cadeias poliméricas do amido, dificultando a sua reorganização e posterior retrogradação [12]. Em contrapartida, para maiores concentrações de ceras, como, apesar da sua natureza lipídica, à temperatura ambiente, as ceras se apresentam no estado sólido, a matriz amido-plasticizante serve de suporte para as ceras, dando origem a materiais com maior resistência à tração [13]. 

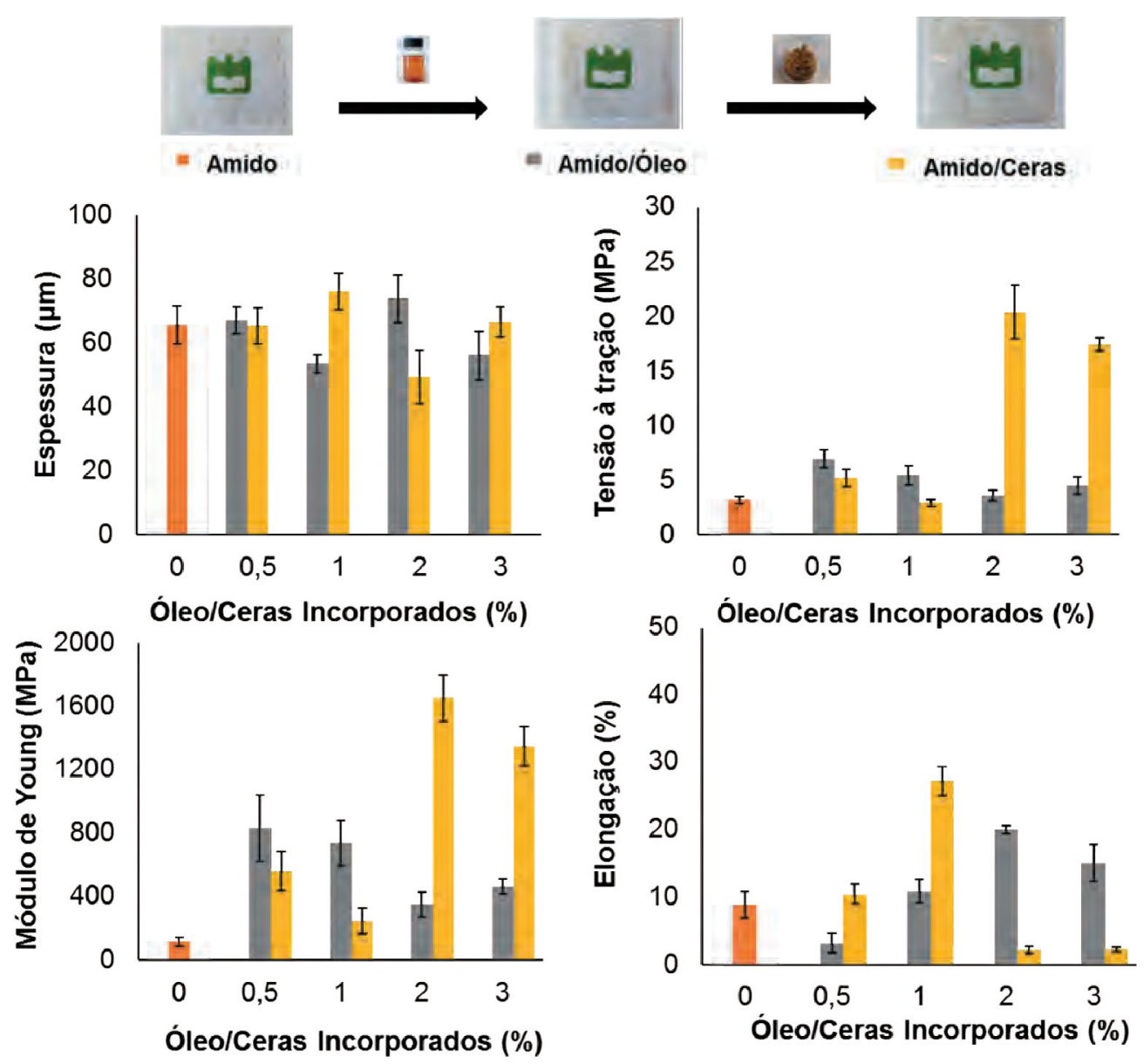

Figura 2 - Bioplásticos à base de amido/óleo/ceras recuperados dos subprodutos da indústria de batata e respetivas propriedades mecânicas.

A hidrofobicidade de cada amostra pode ser medida através da determinação do ângulo de contacto resultante da aplicação de uma gota de água ultrapura na superfície do filme (Figura 3). O valor do ângulo de contacto corresponde ao ângulo medido após estabilização da gota depositada na superfície do filme (cerca de 10-20 segundos). Quanto maior o ângulo de contacto obtido, maior a hidrofobicidade da superfície. A incorporação de óleo ou ceras na formulação conduz ao aumento do valor do ângulo de contacto quando comparado com o filme apenas à base de amido. A lipofilicidade do óleo e das ceras leva a que a sua combinação com uma matriz hidrofílica melhore as propriedades de barreira à água dos filmes à base de amido. Os filmes não apresentaram migração dos compostos hidrofóbicos para a superfície. O uso de uma ultra-agitação através da utilização do ultraturrax poderá ter estabilizado a formulação.

\section{Conclusões}

O projeto POTATOPLASTIC tem revelado que os subprodutos da indústria de batata frita podem ser valorizados através da recuperação de compostos para a produção de bioplásticos. Das águas de lavagem, dos resíduos da fritura e das cascas de batata podem ser recuperados amido, óleo e ceras, respetivamente, com perfil adequado à produção de bioplásticos. A preparação de formulações à base de amido, amido/óleo e amido/ceras permite obter materiais com diferentes propriedades mecânicas e graus de hidrofobici-
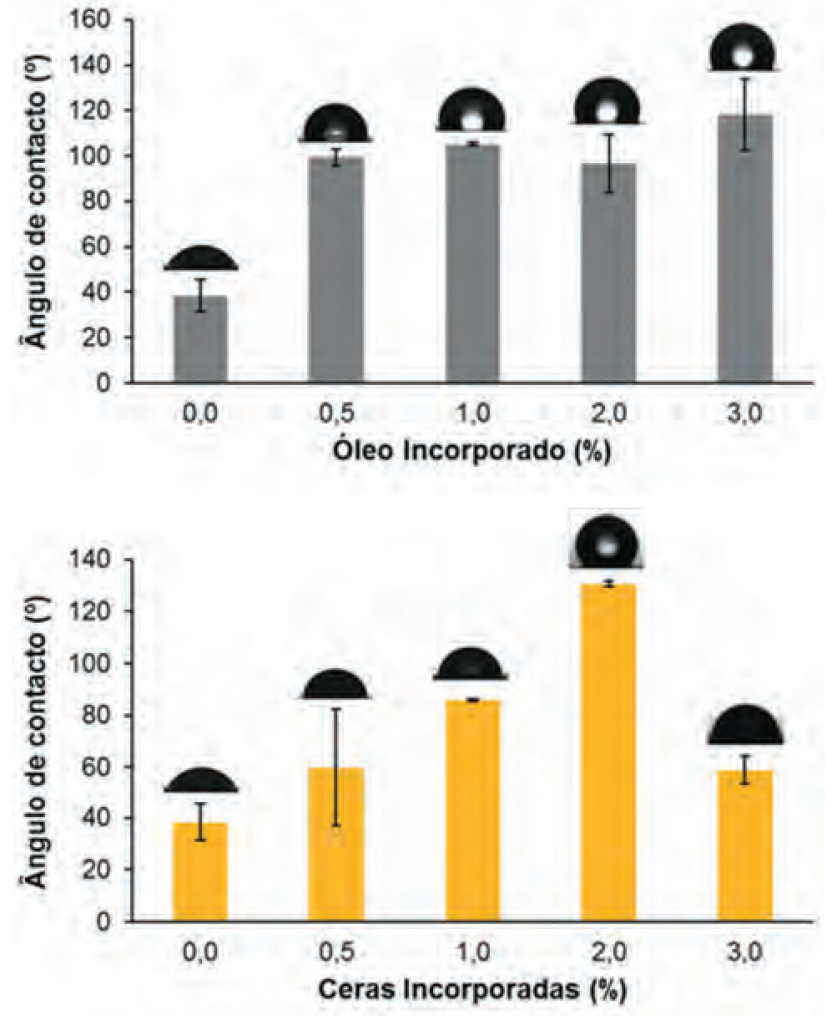

Figura 3 - Ângulo de contacto de uma gota de água aplicada na superfície de filmes bioplásticos à base de amido/óleo/ceras recuperados dos subprodutos da indústria de batata. 
dade, o que demonstra a versatilidade da utilização destes biopolímeros para o desenvolvimento de novos bioplásticos. O próximo desafio consiste na preparação de materiais adequados para o processo de extrusão, com vista à sua transposição ao nível industrial.

\section{Agradecimentos}

Este trabalho foi desenvolvido nas unidades de investigação QOPNA (FCT UID/QUI/00062/2013) e CICECO-Aveiro Institute of Materials, POCI-01-0145-FEDER-007679 (FCT UID/CTM/50011/2013), no âmbito do projeto Copromoção I\&D Empresas intitulado "POTATOPLASTIC - Desenvolvimento de um composto bioplástico biodegradável à base dos subprodutos de batata” (POCI-01-0247-FEDER-017938), financiado pelo Fundo Europeu de Desenvolvimento Regional (FEDER), através do Programa Operacional Competitividade e Internacionalização (POCI). I. Gonçalves, C. Nunes e P. Ferreira agradecem também à FCT a atribuição das bolsas SFRH/BPD/104712/2014, SFRH/BPD/100627/2014 e IF/00300/2015, respetivamente.

\section{Referências}

[1] E.J. North, R.U. Halden, Rev. Environ. Health, 28 (2013) 1-8.
[2] R.J. Slack, J.R. Gronow, N. Voulvoulis, Sci. Total Environ. 337 (2005) 119-137.

[3] S.M. Emadian, T.T. Onay, B. Demirel, Waste Manag. 59 (2017) 526-536.

[4] S. Brockhaus, M. Petersen, W. Kersten, J. Clean. Prod. 127 (2016) 84-95.

[5] P. Morone, V.E. Tartiu, P. Falcone, J. Clean. Prod. 90 (2015) 43-54.

[6] D. Pleissner, Q. Qi, C. Gao, C.P. Rivero, C. Webb, C.S.K. Lin, J. Venus, Biochem. Eng. J. 116 (2016) 3-16.

[7] K. Alvani, X. Qi, R.F. Tester, C.E. Snape, Food Chem. 125 (2011) 958-965.

[8] E. Basiak, A. Lenart, F. Debeaufort, Int. J. Biol. Macromol. 98 (2017) 348-356.

[9] X. Chen, X. Du, P. Chen, L. Guo, Y. Xu, X. Zhou, Carbohydr. Polym. 157 (2017) 637-642.

[10] L. Schreiber, R. Franke, K. Hartmann, Planta 220 (2005) 520-530.

[11] B.M. Szafranek, E.E. Synak, Phytochemistry 67 (2006) 80-90.

[12] D. Kowalczyk, B. Baraniak, Food Hydrocoll. 41 (2014) 195-209.

[13] D. Muscat, R. Adhikari, S. McKnight, Q. Guo, B. Adhikari, J. Food Eng. 119 (2013) 205-219.

Atualidades Científicas

\section{Motor molecular baseado numa estrutura em dupla hélice}

O desenho e síntese de motores moleculares artificiais capazes de executar movimentos rotativos complexos têm atraído grande interesse na comunidade científica no contexto das máquinas moleculares. Por exemplo, a maioria dos motores moleculares preparados até à data consiste em motores rotativos unidirecionais onde um rotor central está ligado a um estator (parte fixa do motor). Ao mesmo tempo, a síntese de oligómeros e polímeros que adotam uma conformação semelhante à da dupla hélice da estrutura do ADN tem sido desafiante para a comunidade científica. Recentemente, estruturas supramoleculares baseadas em ésteres cíclicos de ácidos borónicos foram identificadas como podendo adotar uma conformação semelhante à da dupla hélice da estrutura do ADN e que, devido às suas propriedades dinâmicas, poderiam ser exploradas no contexto das máquinas moleculares.

Investigadores da Universidade de Shimane e da Universidade de Nagoya, Japão, exploraram o uso daqueles sistemas tendo sintetizado boronatos cíclicos com estruturas em dupla hélice. Estudos por difração de raios-X, RMN e dicroísmo circular, além de cálculos teóricos, mostraram que as duplas hélices funcionam como um "rotor molecular”, nas quais as unidades de éster cíclico giram, originando a formação de dois rotâmeros estáveis a baixa temperatura. Ao mesmo tempo, verifica-se também um movimento de inversão helicoidal interconvertível das unidades de boronato cíclico, o que torna, na prática, este sistema capaz de executar dois tipos de movimentos (rotação e inversão helicoidal). Segundo os autores, os seus estudos podem ser úteis no desenvolvimento de rotores moleculares oti-

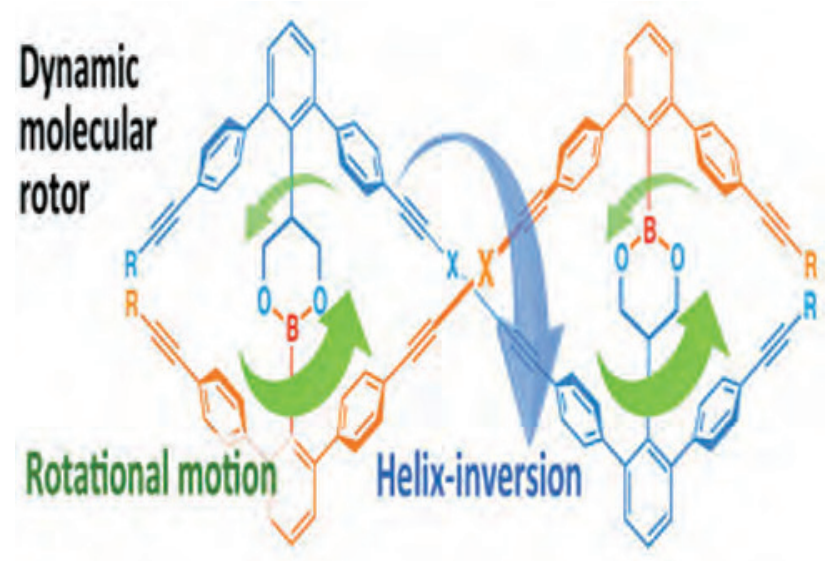
camente ativos pela introdução de substituintes quirais nas unidades do éster borónico.

\section{Fontes:}

Double helices as a molecular rotor, http://www.chemistryviews.org/details/ezine/10477335/Double_Helices_ as_a_Molecular_Rotor.html?elq_mid=16303\&elq_ cid=3941189 (Acedido em 16/04/2017)

H. Iida, K. Ohmura, R. Noda, S. Iwahana, H. Katagiri, N. Ousaka, T. Hayashi, Y. Hijikata, S. Irle, E. Yashima. Double-stranded helical oligomers covalentlybridged by rotary cyclic boronate esters. Chem. Asian J. 12 (2017) 927-935.

Paulo Mendes (pjgm@uevora.pt) 